\author{
Hans Scheffel \\ Hatem Alkadhi \\ André Plass \\ Robert Vachenauer \\ Lotus Desbiolles \\ Oliver Gaemperli \\ Tiziano Schepis \\ Thomas Frauenfelder \\ Thomas Schertler \\ Lars Husmann \\ Jürg Grunenfelder \\ Michele Genoni \\ Philipp A. Kaufmann \\ Borut Marincek \\ Sebastian Leschka
}

\section{Accuracy of dual-source CT coronary angiography: first experience in a high pre-test probability population without heart rate control}

Received: 23 August 2006

Revised: 30 August 2006

Accepted: 31 August 2006

Published online: 19 September 2006

(C) Springer-Verlag 2006

H. Scheffel · H. Alkadhi $(\bowtie)$ •

L. Desbiolles - T. Frauenfelder ·

T. Schertler · L. Husmann

B. Marincek · S. Leschka

Institute of Diagnostic Radiology,

University Hospital Zurich,

Raemistrasse 100,

8091 Zurich, Switzerland

e-mail: hatem.alkadhi@usz.ch

Tel.: +41-1-2553662

Fax: +41-1-2554443

A. Plass $\cdot$ R. Vachenauer

J. Grunenfelder · M. Genoni

Clinic for Cardiovascular Surgery,

Zurich, Switzerland

O. Gaemperli · T. Schepis ·

P. A. Kaufmann

Cardiovascular Center,

University Hospital Zurich,

Zurich, Switzerland

P. A. Kaufmann

Center for Integrative Human

Physiology, University of Zurich,

Zurich, Switzerland
Abstract The aim of this study was to assess the diagnostic accuracy of dualsource computed tomography (DSCT) for evaluation of coronary artery disease (CAD) in a population with extensive coronary calcifications without heart rate control. Thirty patients (24 male, 6 female, mean age $63.1 \pm 11.3$ years) with a high pre-test probability of CAD underwent DSCT coronary angiography and invasive coronary angiography (ICA) within $14 \pm 9$ days. No beta-blockers were administered prior to the scan. Two readers independently assessed image quality of all coronary segments with a diameter $\geq 1.5 \mathrm{~mm}$ using a four-point score (1: excellent to 4: not assessable) and qualitatively assessed significant stenoses as narrowing of the luminal diameter $>50 \%$. Causes of false-positive (FP) and false-negative (FN) ratings were assigned to calcifications or motion artifacts. ICA was considered the standard of reference. Mean body mass index was $28.3 \pm 3.9 \mathrm{~kg} / \mathrm{m}^{2}$ (range $22.4-36.3 \mathrm{~kg} / \mathrm{m}^{2}$ ), mean heart rate during CT was $70.3 \pm 14.2 \mathrm{bpm}$ (range 47-102 bpm), and mean Agatston score was $821 \pm 904$ (range 0 $3,110)$. Image quality was diagnostic (scores $1-3)$ in $98.6 \%(414 / 420)$ of segments (mean image quality score $1.68 \pm 0.75)$; six segments in three patients were considered not assessable $(1.4 \%)$. DSCT correctly identified 54 of 56 significant coronary stenoses. Severe calcifications accounted for false ratings in nine segments (eight FP/one FN) and motion artifacts in two segments (one $\mathrm{FP}$ /one FN). Overall sensitivity, specificity, positive and negative predictive value for evaluating $\mathrm{CAD}$ were $96.4,97.5,85.7$, and 99.4\%, respectively. First experience indicates that DSCT coronary angiography provides high diagnostic accuracy for assessment of CAD in a high pre-test probability population with extensive coronary calcifications and without heart rate control.

Keywords Dual-source computed tomography - Coronary angiography . Coronary stenosis

\section{Introduction}

Recent advances in multi-detector-row computed tomography $(\mathrm{CT})$ technology have continuously improved the quality of non-invasive coronary artery imaging. As a result, various studies have demonstrated a high accuracy of coronary angiography with 64-slice CT for the diagnosis of coronary artery disease (CAD) [1-8]. In particular, the high negative predictive value has made non-invasive coronary angiography using 64-slice CT a modality that 
allows significant coronary stenoses to be reliably excluded. Consequently, the Task Force on the Management of Stable Angina Pectoris of the European Society of Cardiology has recently recommended in their guidelines that CT coronary angiography be performed in patients with stable angina who have a low pre-test probability of $\mathrm{CAD}$, and an inconclusive exercise electrocardiogram (ECG) or stress imaging test [9].

To date, many studies have been published assessing the diagnostic performance of CT coronary angiography with different generations of scanners. Four-slice CT coronary angiography showed limited resolution and required long breath-hold times of nearly $40 \mathrm{~s}$, resulting in a high number of vessel segments that could not be assessed [10]. Sixteenslice CT coronary angiography showed improved diagnostic accuracy because of reduced breath-hold times and an increased temporal and spatial resolution [11, 12]. Sixtyfour-slice CT scanners were the first to allow highly accurate identification of significant coronary artery stenoses [1-8]. However, data analysis in several of these studies could not be performed in all coronary artery segments, with a percentage of non-assessable segments of up to $12 \%$ [2]. The main reasons were severe coronary artery wall calcifications and motion artifacts, the latter occurring especially with higher heart rates. Calcifications are known to complicate the assessment of coronary arteries [10], however, some authors have suggested that substantial coronary calcification should not be used as a reason to defer CT coronary angiography [13].

In order to reduce motion artifacts, the administration of either intravenous or oral beta-blocker medication for heart rate control has been proposed even for 64-slice CT systems $[2-6,8,11,14]$. In most studies, target heart rates for scanning were below 60-65 beats per minute (bpm). Another concept to comply with higher coronary velocities at higher heart rates is the use of multi-segment reconstruction algorithms that combine data from two or more consecutive cardiac cycles to improve temporal resolution at certain heart rates [15]. However, the latter approach relies on a complete periodicity of heart motion, limiting its use in patients with variable heart rates; does not take into account the inter-heartbeat variability of coronary artery position; and requires low pitch factors, prolonging data acquisition times [16]. In line with this, a recent 64-slice CT study demonstrated no significant overall benefit of multi-segment reconstruction with regard to image quality of coronary arteries [17].

The recently introduced dual-source CT (DSCT) scanner is characterized by two $\mathrm{x}$-ray tubes and two corresponding detectors mounted onto the rotating gantry with an angular offset of $90^{\circ}$ [18]. Regarding cardiac imaging capabilities, the new scanner system offers a high temporal resolution of $83 \mathrm{~ms}$ in a mono-segment reconstruction mode. Temporal resolution is independent of the heart rate, which is a major difference from single-source CT systems that rely on multi-segment reconstruction techniques. The first feasi- bility studies have shown promising results with DSCT coronary angiography regarding image quality of coronary arteries, cardiac valves, and left ventricular myocardium independent of the actual heart rate of the patient $[19,20]$. However, to date no study has investigated the diagnostic accuracy of DSCT coronary angiography for the diagnosis of CAD.

The purpose of our study was to determine the performance of DSCT coronary angiography in diagnosing significant stenoses in comparison to invasive coronary angiography (ICA) in a high pre-test probability patient population without heart rate control.

\section{Materials and methods}

Patients

In 30 patients (6 women, 24 men; mean age $63.1 \pm$ 11.3 years; age range 35-86 years) who had undergone ICA for evaluation of suspected CAD, CT coronary angiography was performed within 30 days of catheterization (mean $14 \pm 9$ days). Exclusion criteria for DSCT were allergy to iodine-containing contrast medium, renal insufficiency (creatinine level $>120 \mu \mathrm{mol} / \mathrm{L}$ ), pregnancy, hemodynamic instability, and previous stent graft placement or bypass surgery. Symptoms and cardiovascular risk factors of all patients are reported in Table 1. Based on a previously published clinical pre-test score [21], each patient in this population had a high pre-test probability of CAD. Patients with elevated or irregular heart rates were not excluded from the study. The study protocol was approved by our local ethics committee and all participating patients gave written informed consent.

Table 1 Synopsis of cardiovascular risk factors and symptoms Frequency (\%)

\begin{tabular}{ll} 
Risk factors & \\
Family history & $16(53 \%)$ \\
Obesity & $23(77 \%)$ \\
Dyslipidemia & $18(60 \%)$ \\
Diabetes & $19(63 \%)$ \\
Smoking & $25(83 \%)$ \\
Hypertension & $23(77 \%)$ \\
Symptoms & \\
Angina pectoris & $21(70 \%)$ \\
Probable angina pectoris & $7(23 \%)$ \\
Atypical chest pain & $2(7 \%)$ \\
\hline
\end{tabular}

Risk factors

Dyslipidemia

$23(77 \%)$

Diabetes

$19(63 \%)$

Smoking

$25(83 \%)$

Symptoms

Angina pectoris

$7(23 \%)$

Atypical chest pain

$2(7 \%)$ 


\section{Dual-source CT scan protocol}

All CT examinations were performed on a DSCT scanner (Somatom Definition, Siemens Medical Solutions, Forchheim, Germany). The patients were centrally placed in the scanner to ensure that the entire heart was covered with the smaller field-of-view of the second tube detector array. Irrespective of the individual heart rate and the heart rate variability, no beta-blockers were given prior to the scan. Three patients took oral beta-blockers as part of their baseline medication.

Nonenhanced DSCT for calcium scoring was performed from $1 \mathrm{~cm}$ below the level of the tracheal bifurcation to the diaphragm in a cranio-caudal direction using the following scanning parameters: detector collimation $32 \times 0.6 \mathrm{~mm}$, slice acquisition $64 \times 0.6 \mathrm{~mm}$ by means of a $\mathrm{z}$-flying focal spot, gantry rotation time $330 \mathrm{~ms}$, pitch of $0.2-0.39$ adapted to the heart rate, tube current $80 \mathrm{mAs}$ per rotation, and tube potential $120 \mathrm{kV}$.

Thereafter, all patients received a single dose of $2.5 \mathrm{mg}$ isosorbiddinitrate s. 1. (Isoket, Schwarz Pharma, Monheim, Germany) [22]. After $2 \mathrm{~min}$, the coronary angiography scan was started by continuously injecting a bolus of $80 \mathrm{ml}$ of iodixanol (Visipaque 320, $320 \mathrm{mg} / \mathrm{mL}$, GE Healthcare, Buckinghamshire, UK) followed by $30 \mathrm{ml}$ saline solution into an antecubital vein via an 18-gauge catheter (injection rate $5 \mathrm{~mL} / \mathrm{s}$ ). Contrast agent application was controlled by bolus tracking. A region of interest (ROI; mean diameter $10.1 \pm 5.6 \mathrm{~mm}$, range $7.5-17.0 \mathrm{~mm}$ ) was placed into the aortic root, and image acquisition started $5 \mathrm{~s}$ after the signal attenuation reached the predefined threshold of 100 Hounsfield units (HU). Data acquisition was performed from $1 \mathrm{~cm}$ below the level of the tracheal bifurcation to the diaphragm in a cranio-caudal direction with a detector collimation of $32 \times 0.6 \mathrm{~mm}$, slice acquisition $64 \times 0.6 \mathrm{~mm}$ by means of a z-flying focal spot, gantry rotation time $330 \mathrm{~ms}$, pitch of $0.2-0.43$ adapted to the heart rate, tube current $400 \mathrm{mAs}$ per rotation, and tube potential $120 \mathrm{kV}$. ECGpulsing for radiation dose reduction was applied in all patients. At mean heart rates below $60 \mathrm{bpm}$, full tube current was applied from 60 to $70 \%$, at $61-70 \mathrm{bpm}$ from 50 to $80 \%$, and at heart rates above 70 from 30 to $80 \%$ of the RR-interval. The electrocardiogram (ECG) was digitally recorded during data acquisition and was stored with the unprocessed CT dataset.

\section{Dual-source CT image reconstruction}

A retrospective gating technique was used to synchronize the data reconstruction with the ECG signal. A monosegment reconstruction algorithm that uses the data from a quarter rotation of both detectors was used for image reconstruction [18]. In each patient, images were first reconstructed at 60 and $70 \%$ of the RR-interval. If considered necessary, additional images were reconstructed in $5 \%$ steps of the RR interval within the full tube current window. In case of irregular heart rates, the temporal variability in the reconstruction phase was compensated by manual ECG editing. In case of premature heart beats, the temporal window past the premature heart beat was deleted, and the next diastolic window was filled with one to three temporal windows to avoid data gaps. All ECG editing was performed by one experienced cardiovascular radiologist not involved in data read-out. For calcium scoring, non-overlapping images with a slice width of $3 \mathrm{~mm}$ were reconstructed using a medium-sharp convolution kernel (B35f). For DSCT coronary angiography, images were reconstructed from the contrast-enhanced DSCT scan with a slice thickness of $0.75 \mathrm{~mm}$, a reconstruction increment of $0.5 \mathrm{~mm}$, and using a medium soft-tissue convolution kernel (B26f). Depending on the individual anatomy, the reconstructed field-of-view (FoV) was adjusted to encompass the heart exactly (mean FoV $167 \pm 19 \mathrm{~mm}$; range 131 $187 \mathrm{~mm}$; image matrix $512 \times 512$ pixels). After removing patient and ECG information, all reconstructed images were transferred to a dedicated workstation (Wizard, Siemens Medical Solutions) equipped with dedicated cardiac post-processing software (Syngo Circulation, Siemens Medical Solutions).

\section{Dual-source CT data analysis}

The mean Agatston score was calculated for each patient from the non-enhanced DSCT data with a detection threshold of $130 \mathrm{HU}$ by using semi-automated software (Syngo Calcium Scoring, Siemens Medical Solutions).

For analysis of DSCT coronary angiography data, coronary arteries were segmented according to the guidelines of the American Heart Association [23]. The right coronary artery (RCA) was defined to include segments $1-4$, the left main and left anterior descending artery (LMLAD) to include segments 5-10, and the left circumflex artery (LCX) to include segments 11-15. The intermedial artery was designated as segment 16 , if present. All segments with a diameter of at least $1.5 \mathrm{~mm}$ at their origin were included. Diameter measurements were performed with an electronic caliper tool. Segments distal to an occluded vessel were excluded from analysis.

All reconstructed images were evaluated and classified by two independent readers using axial source images, multi-planar reformations (MPR), and thin-slab maximum intensity projections (MIP) on a per-segment basis. Both readers semi-quantitatively assessed the image quality of each coronary segment on a four-point ranking scale as previously published [1]: 1, excellent (no artifacts, unrestricted evaluation); 2, good (minor artifacts, good diagnostic quality); 3, adequate (moderate artifacts, still acceptable and diagnostic), and 4, not assessable (severe artifacts impairing accurate evaluation). Causes of image degradation were noted by both observers as arterial wall 
calcifications, motion artifacts, or others. Motion artifacts were defined as any impairment of image quality caused by vessel movement resulting in blurred or doubled vessel contours. Other reasons included low vessel opacification, low signal-to-noise ratio, and disturbing adjacent structures such as contrast-enhanced cardiac veins.

In addition, both readers assessed all coronary artery segments for the presence of hemodynamically significant stenoses. Significant stenosis was defined as narrowing of the coronary luminal diameter exceeding $50 \%$. The vessel diameters were measured on reconstructions perpendicularly oriented to the vessel course.

For any disagreement in data analysis, consensus agreement was achieved.

\section{Invasive coronary angiography}

ICA was performed according to standard techniques, and multiple views were stored on a CD-ROM. The angiograms were evaluated by one experienced observer who was blinded to the results of the DSCT coronary angiography. Coronary artery segments were defined according to the same guidelines mentioned above [23]. Each vessel segment was scored as being significantly stenosed, defined as a diameter reduction of $>50 \%$ or not. Coronary artery analysis was performed in all vessels with a luminal diameter of at least $1.5 \mathrm{~mm}$, excluding those vessels distal to complete occlusions.

\section{Statistical analysis}

Statistical analysis was performed using commercially available software (SPSS 12.0, SPSS, Chicago, IL, USA). Quantitative variables were expressed as mean \pm SD and categorical variables as frequencies or percentages. In a subanalysis, patients were subdivided into mean heart rates of $<70 \mathrm{bpm}$ and $\geq 70 \mathrm{bpm}$ and into Agatston scores of $<400$ and $\geq 400$. We took into account the clustered nature of the data (i.e., the fact that there were not 420 independent vessel segments but instead clusters of segments in 30 patients). Inter-observer agreements for image quality readout and assessment of significant coronary artery stenoses were interpreted according to the guidelines of Landis and Koch [24] using the clustered data. Sensitivity, specificity, positive predictive value, and negative predictive value were calculated from chi-squared tests of contingency, and the $95 \%$ confidence intervals (CI) were calculated from binomial expression on a per-segment basis. Because of the interdependencies between different segments, the statistics were also calculated on a per-patient basis (presence of at least one significant coronary artery stenosis or absence of any significant stenosis in each patient). ICA was considered the standard of reference.

\section{Results}

DSCT and ICA were successfully performed in all patients without complications. The DSCT protocol was well tolerated by all patients, and all were able to hold their breath during data acquisition. The average HR during scanning was $70.3 \pm 14.2 \mathrm{bpm}$ (range $47-102 \mathrm{bpm}$ ). Seventeen patients $(56.7 \%)$ had a heart rate below $70 \mathrm{bpm}$ (mean $59.7 \pm 5.9 \mathrm{bpm}$, range $47-66 \mathrm{bpm}$ ), while 13 patients (43.3\%) had a heart rate of $\geq 70 \mathrm{bpm}$ (mean $84.2 \pm 8.4 \mathrm{bpm}$, range $72-102 \mathrm{bpm})$.

Prevalance of coronary artery stenosis and calcium score

A total of 56 coronary artery stenoses with a luminal narrowing of more than $50 \%$ in diameter were identified in 15 patients $(50.0 \%)$ using ICA. Single-vessel disease was present in $13.3 \%$ (4/30), two-vessel disease in $10.0 \%$ (3/30), and three-vessel disease in $26.7 \%$ (8/30). Significant coronary artery stenoses could be excluded in 15 patients $(50.0 \%)$.

Calcified vessel wall deposits were present in 24 patients $(80 \%)$. Fourteen of these patients $(58.3 \%)$ had significant coronary artery stenoses, while 10 patients $(41.7 \%)$ had calcifications without significant stenoses. The mean Agatston score was $821 \pm 904$ (range 0-3,110). Agatston score was $<400$ in 15 patients $(50 \%$, mean score $85 \pm 118)$ and $\geq 400$ in the other 15 patients $(50 \%$, mean score $1,483 \pm$ $893)$.

Image quality and image reconstruction intervals with DSCT

A total of 420 coronary artery segments with a diameter $\geq 1.5 \mathrm{~mm}$ were evaluated (11 segments were missing because of anatomical variants, 26 segments had a diameter less than $1.5 \mathrm{~mm}$ at their origin, and 9 segments were distal to an occluding stenosis). An intermedial artery was present in 16 patients $(53.3 \%)$. Inter-observer agreement for image quality rating using clustered data was moderate (kappa $=0.68)$. The initially used reconstruction time-points of 60 and $70 \%$ provided excellent image quality (score 1) in $21.9 \%(92 / 420)$ and $60.2 \%(253 / 420)$, respectively, while additional reconstructions were necessary in $17.9 \%$ (75/420) to improve image quality. Whereas in the subgroup of patients with heart rates $\geq 70 \mathrm{bpm}$ additional reconstructions at 30 to $50 \%$ were considered necessary in $27.4 \%(48 / 175)$, in patients with heart rates $<70 \mathrm{bpm}$, the 60 and $70 \%$ interval provided excellent image quality (score 1) in $90.2 \%$ of the segments [30.2\% (74/245) and $60.0 \%(147 / 245)$, respectively].

Using the individual reconstruction interval with best image quality for each segment, image quality was 
excellent (score 1) in $47.4 \%$ of the coronary segments (199/420), good (score 2) in 37.9\% (159/420), adequate (score 3 ) in $13.3 \%(56 / 420)$, and poor/not assessable in $1.4 \%(6 / 420)$. Non-assessable (score 4$)$ coronary artery segments (segment $4, n=2$; segment $8, n=1$; segment 10 , $n=2$; and segment $14, n=1$ ) were present in three patients with heart rates of 72,77 , and $86 \mathrm{bpm}$, and Agatston scores of 30,616 , and 0 , respectively.

In the 221 segments with image quality rated other than excellent (score 1), reasons for impaired image quality were arterial wall calcifications in $48.9 \%(108 / 221)$, motion artifacts in $46.6 \%(103 / 221)$, or other in $4.5 \%$ of segments (10/221; low signal-to-noise ratio, $n=5$; low vessel opacification, $n=4$; overlaying adjacent structures, $n=1$ ). At heart rates $\geq 70 \mathrm{bpm}$, minor to moderate motion artifacts accounted for $68.2 \%$ (73/107) of image quality impairment, while in patients with an Agatston score $\geq 400$, severe arterial wall calcifications were mainly responsible for decreased image quality [76.4\% (94/123)].

\section{Diagnostic accuracy of DSCT in comparison to ICA}

The kappa value for coronary artery stenosis detection with DSCT was 0.83 indicating a good inter-observer agreement. DSCT coronary angiography correctly recognized 54 of the 56 significant stenoses (96.4\%) detected with ICA. For both readers, nine false-positive (FP) and two falsenegative (FN) ratings occurred with DSCT coronary angiography. Causes of FP and FN ratings were massive calcifications in nine segments (eight FP, one FN) and motion artifacts in two segments (one FP, one FN). The two FN ratings occurred in segments 8 and 9 . Examples of three patients with suspicion of CAD who underwent DSCT coronary angiography are provided in Figs. 1, 2, and 3.

On a per-segment analysis, overall sensitivity was $96.4 \%$ (54/56; 95\% CI: 87.7-99.6), specificity was $97.5 \%$ (355/364; 95\% CI: 95.4-98.9), positive predictive value was $85.7 \%(54 / 63 ; 95 \%$ CI: $74.6-93.3)$, and negative predictive value was $99.4 \%$ (355/357; 95\% CI: 98.0-99.9). Table 2 summarizes demographic data, overall image quality, and diagnostic accuracy of DSCT.

On a per-patient analysis, sensitivity was $93.3 \%(14 / 15$; 95\% CI: $68.1-99.8)$, specificity was $100 \%(15 / 15 ; 95 \%$ CI: 78.2-100), positive predictive value was $100 \%(14 / 14$; 95\% CI: $76.8-100)$, and negative predictive value was 93.8\% (15/16; 95\% CI: 69.8-99.8).

In both heart-rate subgroups, diagnostic accuracy for the assessment of coronary artery stenosis was similar and the rate of false ratings was comparable (one FN and six FP at heart rates $<70 \mathrm{bpm}$; one FN and three FP at heart rates $\geq 70 \mathrm{bpm}$ ). In contrast, the subgroup of patients with an Agatston score $\geq 400$ included more false ratings (two FN and eight FP) than the subgroup of patients with an Agatston score $<400$ (zero FN and one FP). Consequently, sensitivity and specificity were worse in severely calcified vessels (see Table 2).
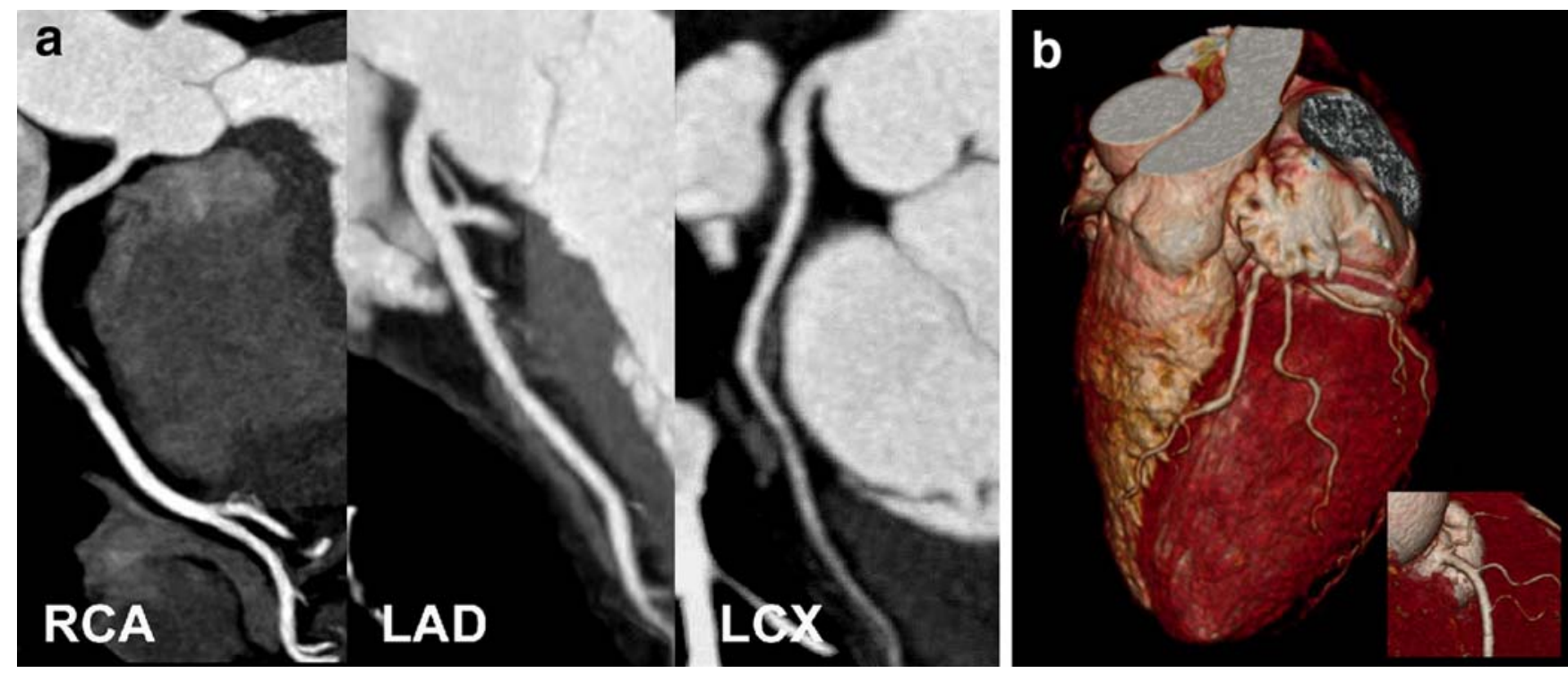

Fig. 1a, b Dual-source CT coronary angiography in a 62-year-old woman with suspected coronary artery disease (mean heart rate during scanning $76 \mathrm{bpm}$, Agatston score 0). a Curved thin-slab maximum-intensity projections through the centerline of the right coronary $(R C A)$, left anterior descending $(L A D)$, and left circumflex artery $(L C X)$. Slight blurring of the mid-RCA and mid-LAD rendered image quality as good (score 2) in these segments, while image quality was rated excellent (score 1) in all other segments. Coronary artery disease could be reliably excluded in this patient. b Volume-rendered image of the left coronary arteries and of the proximal RCA (insert) demonstrates accurate depiction of the coronary artery tree 


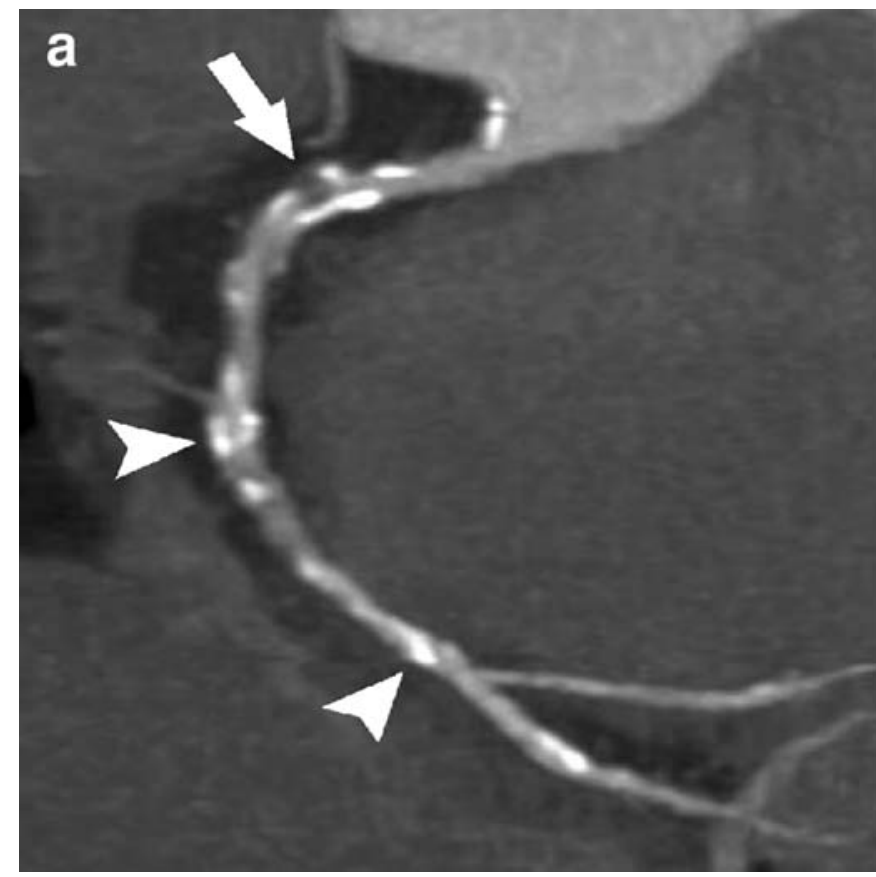

Fig. 2a, b Dual-source CT coronary angiography in a 69-year-old man with suspected coronary artery disease (mean heart rate during scanning $77 \mathrm{bpm}$, Agatston score 1,316). a Curved-planar reconstruction of the right coronary artery demonstrates high-grade coronary artery stenosis of the proximal segment (arrow) and non-

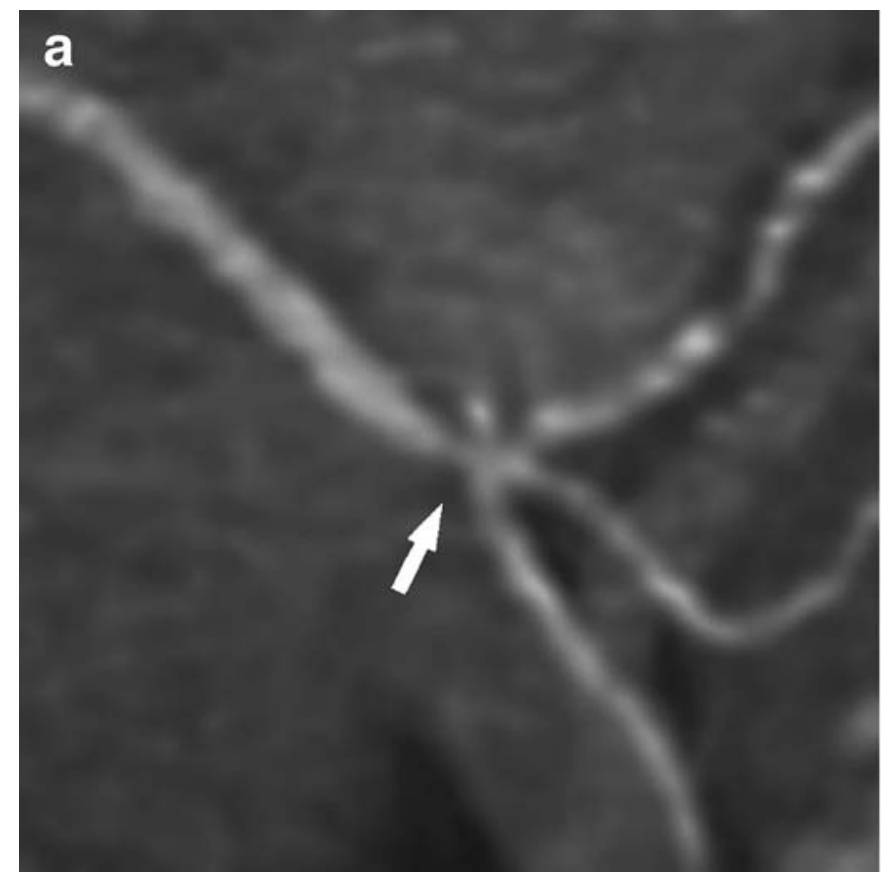

Fig. 3a, b Dual-source CT scan in a 73-year-old man with suspected coronary artery disease (mean heart rate during scanning 86 bpm, Agatston score 259). a Thin-slab maximum-intensity projection shows stenosis of the distal right coronary artery near the origin of the posterior descending artery. This stenosis was rated significant (i.e., $>50 \%$ luminal diameter narrowing) by both readers.

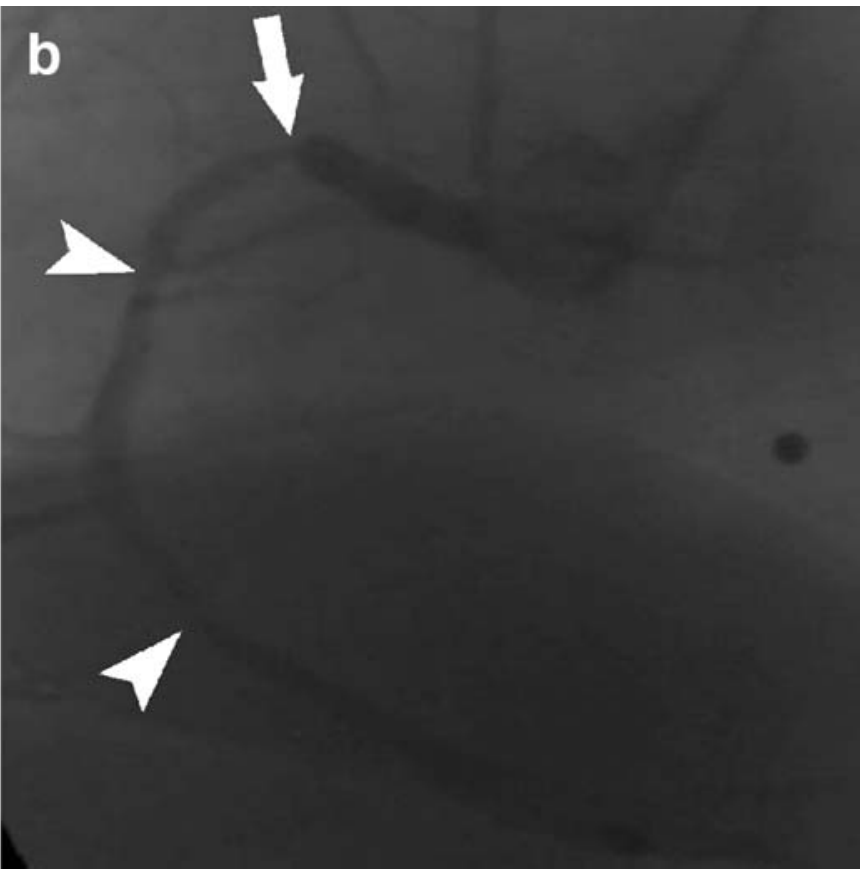

significant stenoses of the mid and distal segments (arrowheads). b Invasive coronary angiography in a left anterior oblique projection $\left(45^{\circ}\right)$ confirms significant stenosis of the proximal segment of the right coronary artery (arrow) and non-significant stenoses of the mid and distal segments (arrowheads)

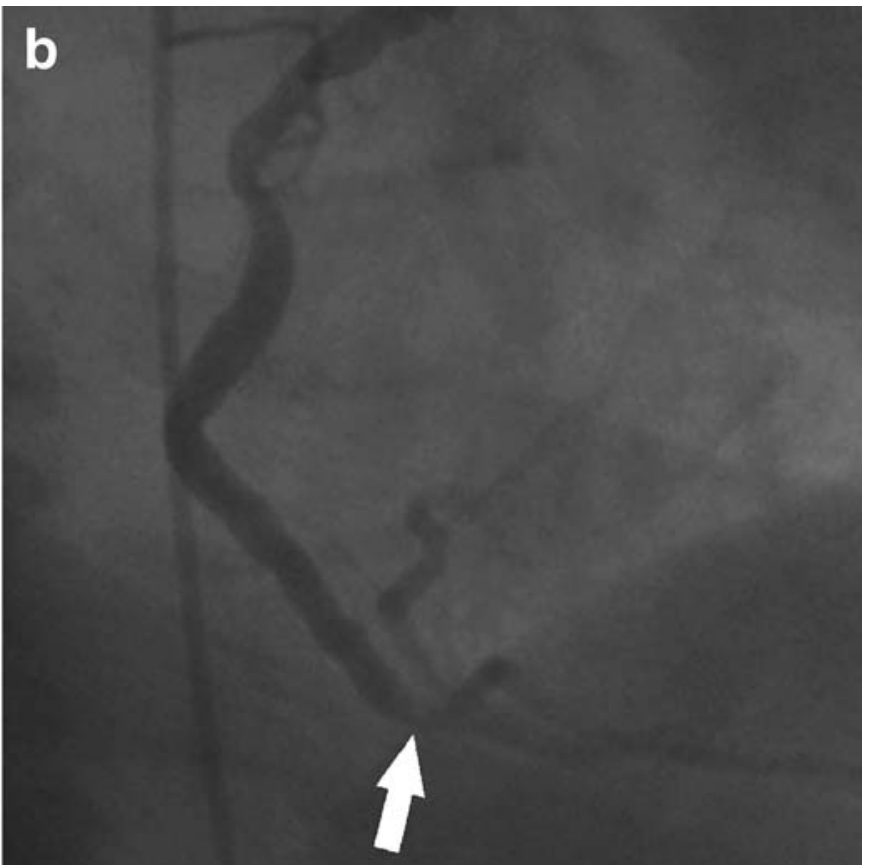

b Invasive coronary angiography in a right anterior oblique projection shows irregular luminal narrowing in the distal right coronary artery that was qualitatively graded as less than $50 \%$ luminal diameter narrowing, resulting in a false positive diagnosis in DSCT coronary angiography 
Table 2 Demographic data, overall image quality, and diagnostic accuracy

\begin{tabular}{|c|c|c|c|c|c|}
\hline & \multirow[b]{2}{*}{ Total } & \multicolumn{2}{|l|}{ Mean heart rate } & \multicolumn{2}{|l|}{ Agatston score } \\
\hline & & $<70 \mathrm{bpm}$ & $\geq 70 \mathrm{bpm}$ & $<400$ & $\geq 400$ \\
\hline No. of patients & 30 & 17 & 13 & 15 & 15 \\
\hline Age (years) & $63.1 \pm 11.3$ & $63.2 \pm 10.1$ & $62.9 \pm 13.3$ & $62.8 \pm 13.7$ & $63.4 \pm 8.9$ \\
\hline Male/female & $24 / 6$ & $15 / 2$ & $9 / 4$ & $10 / 5$ & $14 / 1$ \\
\hline BMI $\left(\mathrm{kg} / \mathrm{m}^{2}\right)$ & $28.3 \pm 3.9$ & $28.9 \pm 4.3$ & $27.6 \pm 3.5$ & $28.1 \pm 3.5$ & $28.5 \pm 4.4$ \\
\hline Mean heart rate (bpm) & $70.3 \pm 14.2$ & $59.7 \pm 5.9$ & $84.2 \pm 8.4$ & $70.6 \pm 13.7$ & $70.0 \pm 15.1$ \\
\hline Agatston score & $821 \pm 904$ & $901 \pm 991$ & $674 \pm 780$ & $85 \pm 118$ & $1,483 \pm 893$ \\
\hline Overall image quality ${ }^{\mathrm{a}}$ & $1.68 \pm 0.75$ & $1.60 \pm 0.73$ & $1.81 \pm 0.77$ & $1.59 \pm 0.75$ & $1.79 \pm 0.75$ \\
\hline Score 1 & $47.4 \%(199 / 420)$ & $51.8 \%(131 / 245)$ & $38.9 \%(68 / 175)$ & $54.2 \%(116 / 214)$ & $40.3 \%(83 / 206)$ \\
\hline Score 2 & $37.9 \%(159 / 420)$ & $32.4 \%(82 / 245)$ & $44.0 \%(77 / 175)$ & $35.5 \%(76 / 214)$ & $40.3 \%(83 / 206)$ \\
\hline Score 3 & $13.3 \%(56 / 420)$ & $11.9 \%(30 / 245)$ & $14.9 \%(26 / 175)$ & $7.5 \%(16 / 214)$ & $19.4 \%(40 / 206)$ \\
\hline Score 4 & $1.4 \%(6 / 420)$ & $0.8 \%(2 / 245)$ & $2.2 \%(4 / 175)$ & $2.8 \%(6 / 214)$ & - \\
\hline Sensitivity & $96.4 \%(54 / 56)$ & $97.2 \%(35 / 36)$ & $95.0 \%(19 / 20)$ & $100 \%(5 / 5)$ & $96.1 \%(49 / 51)$ \\
\hline $95 \% \mathrm{CI}$ & $87.7-99.6$ & $85.5-99.9$ & $75.1-99.9$ & $47.8-100$ & $86.5-99.5$ \\
\hline Specificity & $97.5 \%(355 / 364)$ & $97.1 \%(203 / 209)$ & $98.0 \%(152 / 155)$ & $99.5 \%(208 / 209)$ & $94.8 \%(147 / 155)$ \\
\hline $95 \% \mathrm{CI}$ & $95.4-98.9$ & $94.2-98.8$ & $94.5-99.6$ & $97.4-100$ & $90.1-97.8$ \\
\hline PPV & $85.7 \%(54 / 63)$ & $85.4 \%(35 / 41)$ & $86.4 \%(19 / 22)$ & $83.3 \%(5 / 6)$ & $86.0 \%(49 / 57)$ \\
\hline $95 \% \mathrm{CI}$ & $74.6-93.3$ & $70.8-94.4$ & $65.1-97.1$ & $35.9-99.6$ & $74.2-93.7$ \\
\hline NPV & $99.4 \%(355 / 357)$ & $99.5 \%(203 / 204)$ & $98.8 \%(152 / 153)$ & $100 \%(208 / 208)$ & $98.7 \%(147 / 149)$ \\
\hline $95 \% \mathrm{CI}$ & $98.0-99.9$ & $97.3-100$ & $96.4-100$ & $98.2-100$ & $95.2-99.8$ \\
\hline
\end{tabular}

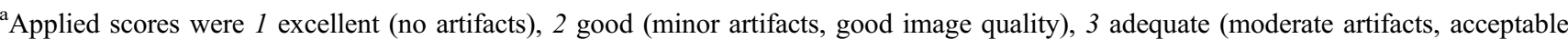
image quality), and 4 not assessable (severe artifacts impairing image evaluation). A score of 1-3 was considered acceptable for diagnosis. $B M I$ Body mass index, $C I$ confidence interval, $P P V$ positive predictive value, $N P V$ negative predictive value

\section{Discussion}

Four main conclusions can be drawn from this study. First, DSCT coronary angiography provides a high diagnostic accuracy for the evaluation of CAD. Second, this high diagnostic performance of DSCT could be achieved in a patient population with extensive calcifications and in whom no heart rate control using beta blocker medication prior to CT was performed. Third, taking into account these circumstances, only six (1.4\%) segments had to be excluded from data analysis, all in the distal part of the coronary artery tree with small vessel diameters. Fourth, false ratings were primarily due to severe vessel wall calcifications rather than motion artifacts.

Temporal resolution better than $100 \mathrm{~ms}$ in combination with submillimeter isotropic spatial resolution and examination times no longer than $10 \mathrm{~s}$ are considered prerequisites for successful implementation of cardiac CT into routine clinical algorithms [18]. DSCT scanners with 0.33 -s gantry rotation time and $32 \times 0.6 \mathrm{~mm}$ collimation in combination with double z-sampling (i.e., simultaneous acquisition of 64 overlapping $0.6-\mathrm{mm}$ slices) fulfill these requirements.

Early feasibility studies confirmed the technical capacity of DSCT to provide diagnostic image quality of coronary arteries in patients with high heart rates $[19,20]$. This study is, to the best of our knowledge, the first to demonstrate a high diagnostic accuracy of DSCT coronary angiography for the diagnosis of CAD in comparison to ICA. By including a patient population with a high prevalence of coronary calcification and without heart rate control during scanning, we found an overall sensitivity of $96.4 \%$ and specificity of $97.5 \%$ for the detection of significant coronary stenoses. These results are comparable to those previously reported with 64-slice CT; however, betablocker administration for strict heart rate control was needed in those studies to compensate for motion artifacts. By including patients with heart rates up to $102 \mathrm{bpm}$, we found minor to moderate vessel wall blurring due to motion artifacts to be present at high heart rates; however, only $1.4 \%$ of all segments had to be excluded from analysis because they were non-diagnostic.

Further confirmation of our preliminary results in larger patient populations may broaden the clinical indications for CT coronary angiography from a modality that is recommended for ruling-out CAD in patients with a low pre-test probability [9] to use in populations with intermediate- or even high pre-test probabilities of the disease. This might also include the implementation of CT coronary angiography as a tool for preoperative planning before cardiac bypass surgery [25] and as a gatekeeper for invasive angiography in patients referred for cardiac valve surgery [26].

Our patient population had a high prevalence of coronary wall calcification, with a mean Agatston score of 821 - being higher than the $75 \%$ percentile in an age- 
and gender-matched control population [27]. With 64-slice CT, Raff et al. [2] reported a considerable decline in diagnostic accuracy in patients with Agatston scores $>400$ with a sensitivity of $93 \%$, a specificity of $67 \%$, and positive and negative predictive values of 93 and $67 \%$, respectively. Using the same cut-off Agatston score, we found a sensitivity of $96 \%$, a specificity of $95 \%$, and positive and negative predictive values of 86 and $99 \%$, respectively. Considering that the spatial resolution of DSCT is the same as that of the single-source 64-slice CT scanner, this apparent difference in calcification-dependency could indicate that the blooming artifact of severely calcified vessel walls may be sometimes superimposed by additional motion artifacts.

We acknowledge the following study limitations. First, we included a relatively small group of only 30 patients. Certainly, future studies with larger patient populations are needed to confirm our first experience. Second, our study patients were a high pre-test probability population, which may have resulted in an overestimation of the ability of DSCT to detect and to rule-out stenoses. In particular the predictive value of positive and negative diagnostic tests is known to be strongly influenced by disease prevalence and pre-test probability. Third, we did not apply the multisegment reconstruction mode, which possibly may further improve the image quality at elevated heart rates. Fourth, CT coronary angiography is associated with substantial irradiation to the patient. However, using the DSCT system allowed the variable use of ECG-pulsing for dose-saving purposes [28] in all of our patients. In the protocol applied in this study, the ECG-pulsing window width was chosen according to the mean heart rate during scanning, i.e., a relatively narrow window width at low and a larger window width at higher heart rates. As previously performed for 64-slice CT [17, 29], studies analyzing optimum reconstruction intervals for DSCT coronary angiography are mandatory to reduce the width of the pulsing window and the applied radiation dose further. Finally, the ability to use the two x-ray tubes simultaneously with different voltages to improve plaque composition characterization [30] and thus potentially to improve the accuracy of stenosis detection has not been investigated.

\section{Conclusion}

First experience indicates that DSCT coronary angiography provides high diagnostic accuracy for assessment of CAD in a high pre-test probability population with extensive coronary calcifications and without heart rate control. Further studies are needed to confirm our results in appropriate clinical settings with larger patient populations.

Acknowledgement This research has been supported by the National Center of Competence in Research, Computer-Aided and Image-Guided Medical Interventions of the Swiss National Science Foundation.

\section{References}

1. Leschka $S$ et al. (2005) Accuracy of MSCT coronary angiography with 64slice technology: first experience. Eur Heart J 26:1482-1487

2. Raff GL, Gallagher MJ, O’Neill WW, Goldstein JA (2005) Diagnostic accuracy of noninvasive coronary angiography using 64-slice spiral computed tomography. J Am Coll Cardiol 46:552-557

3. Leber AW et al. (2005) Quantification of obstructive and nonobstructive coronary lesions by 64 -slice computed tomography: a comparative study with quantitative coronary angiography and intravascular ultrasound. J Am Coll Cardiol 46:147-154

4. Mollet NR et al. (2005) High-resolution spiral computed tomography coronary angiography in patients referred for diagnostic conventional coronary angiography. Circulation 112:2318-2323
5. Pugliese F et al. (2006) Diagnostic accuracy of non-invasive 64-slice CT coronary angiography in patients with stable angina pectoris. Eur Radiol 16:575-582

6. Nikolaou K et al. (2006) Accuracy of 64-MDCT in the diagnosis of ischemic heart disease. AJR Am J Roentgenol 187:111-117

7. Ehara M et al. (2006) Diagnostic accuracy of 64-slice computed tomography for detecting angiographically significant coronary artery stenosis in an unselected consecutive patient population: comparison with conventional invasive angiography. Circ J 70:564-571

8. Ropers D et al. (2006) Usefulness of multidetector row spiral computed tomography with $64-\times 0.6-\mathrm{mm}$ collimation and $330-\mathrm{ms}$ rotation for the noninvasive detection of significant coronary artery stenoses. Am J Cardiol 97:343-348
9. Fox K et al. (2006) Guidelines on the management of stable angina pectoris. Executive summary: the Task Force on the Management of Stable Angina Pectoris of the European Society of Cardiology. Eur Heart J 27:1341-1381

10. Kuettner A et al. (2004) Diagnostic accuracy of multidetector computed tomography coronary angiography in patients with angiographically proven coronary artery disease. J Am Coll Cardiol 43:831-839

11. Mollet NR et al. (2004) Multislice spiral computed tomography coronary angiography in patients with stable angina pectoris. J Am Coll Cardiol 43:2265-2270

12. Kuettner A et al. (2004) Noninvasive detection of coronary lesions using 16detector multislice spiral computed tomography technology: initial clinical results. J Am Coll Cardiol 44:1230 1237 
13. Cademartiri $F$ et al. (2005) Impact of coronary calcium score on diagnostic accuracy for the detection of significant coronary stenosis with multislice computed tomography angiography. Am J Cardiol 95:1225-1227

14. Ropers D et al. (2003) Detection of coronary artery stenoses with thin-slice multi-detector row spiral computed tomography and multiplanar reconstruction. Circulation 107:664666

15. Flohr T, Ohnesorge B (2001) Heart rate adaptive optimization of spatial and temporal resolution for electrocardiogram-gated multislice spiral CT of the heart. J Comput Assist Tomogr 25:907923

16. Flohr TG, Schaller S, Stierstorfer K, Bruder H, Ohnesorge BM, Schoepf UJ (2005) Multi-detector row CT systems and image-reconstruction techniques. Radiology 235:756-773

17. Wintersperger BJ et al. (2006) Image quality, motion artifacts, and reconstruction timing of 64-slice coronary computed tomography angiography with 0.33 -second rotation speed. Invest Radiol 41:436-442
18. Flohr TG et al. (2006) First performance evaluation of a dual-source CT (DSCT) system. Eur Radiol 16:256268

19. Achenbach S et al. (2006) Contrastenhanced coronary artery visualization by dual-source computed tomographyinitial experience. Eur J Radiol 57:331335

20. Johnson TR et al. (2006) Dual-source CT cardiac imaging: initial experience. Eur Radiol 16(7):1409-1415

21. Morise AP, Haddad WJ, Beckner D (1997) Development and validation of a clinical score to estimate the probability of coronary artery disease in men and women presenting with suspected coronary disease. Am J Med 102:350356

22. Dewey M, Hoffmann H, Hamm B (2006) Multislice CT coronary angiography: effect of sublingual nitroglycerine on the diameter of coronary arteries. Rofo 178:600-604

23. Austen WG et al. (1975) A reporting system on patients evaluated for coronary artery disease. Report of the Ad Hoc Committee for Grading of Coronary Artery Disease, Council on Cardiovascular Surgery, American Heart Association. Circulation 51:5-40

24. Landis JR, Koch GG (1977) The measurement of observer agreement for categorical data. Biometrics 33:159174
25. Plass A et al. (2006) Coronary artery imaging with 64-slice computed tomography from cardiac surgical perspective. Eur J Cardiothorac Surg 30:109-116

26. Gilard M et al. (2006) Accuracy of multislice computed tomography in the preoperative assessment of coronary disease in patients with aortic valve stenosis. J Am Coll Cardiol 47:2020-2024

27. Hoff JA, Chomka EV, Krainik AJ, Daviglus M, Rich S, Kondos GT (2001) Age and gender distributions of coronary artery calcium detected by electron beam tomography in 35,246 adults. Am J Cardiol 87:1335-1339

28. Jakobs TF et al. (2002) Multislice helical CT of the heart with retrospective ECG gating: reduction of radiation exposure by ECG-controlled tube current modulation. Eur Radiol 12:1081-1086

29. Leschka S et al. (2006) Optimal image reconstruction intervals for non-invasive coronary angiography with 64 slice CT. Eur Radiol 16:1964-1972

30. Boll DT, Hoffmann MH, Bossert AS, Aschoff AJ, Fleiter TR (2005) New frontiers in coronary plaque imaging and characterization: polychromatic dual energy multidetector CT with histopathologic correlation. Radiology Suppl:SSD08-09 